Musées, Patrimoine et Culture scientifiques et techniques

$187 \mid 2020$

janvier-février 2020

\title{
Rendre aux arbres la place que l'anthropocentrisme leur a pris
}

\section{Marie Perennes et Samuel Cordier}

\section{OpenEdition \\ Journals}

Édition électronique

URL : http://journals.openedition.org/ocim/3552

DOI : 10.4000/ocim.3552

ISSN : 2108-646X

Éditeur

OCIM

Édition imprimée

Date de publication : 1 janvier 2020

Pagination : 36-41

ISSN : 0994-1908

Référence électronique

Marie Perennes et Samuel Cordier, "Rendre aux arbres la place que l'anthropocentrisme leur a pris »,

La Lettre de I'OCIM [En ligne], 187 | 2020, mis en ligne le 01 janvier 2021, consulté le 25 janvier 2021

URL : http://journals.openedition.org/ocim/3552 ; DOI : https://doi.org/10.4000/ocim.3552

Ce document a été généré automatiquement le 25 janvier 2021.

Tous droits réservés 


\title{
Rendre aux arbres la place que l'anthropocentrisme leur a pris
}

\author{
Marie Perennes et Samuel Cordier
}

Détail de l'affiche de l'exposition Nous les Arbres, basée sur l'œuvre $A$ picturesque voyage through Brazil \#37, 2015 de Cássio Vasconcellos.



(C) Cássio Vasconcellos 
SAMUEL CORDIER : On se rappelle de l'exposition Le grand orchestre des animaux, avec Bernie Krause. Ce n'est donc pas la première fois que vous traitez le thème de la place de l'Homme dans le monde vivant à la Fondation Cartier?

Marie Perennes : Par le biais de nos expositions, nous portons notre regard sur des questions majeures d'actualité, et plus particulièrement sur les questions écologiques. Ainsi, la programmation de la Fondation aborde depuis plusieurs années des questions comme celles de la nature, de l'environnement ou du dérèglement climatique. Elle questionne tout ce qui touche à l'impact et la place de l'Homme au sein de la communauté du vivant. Nous avons en effet travaillé sur le thème de la destruction du monde animal, notamment avec une installation comme celle du Grand orchestre des animaux, en 2017. Nous avons également consacré plusieurs projets aux questions des migrations, de la déforestation, de la disparition des cultures autochtones, avec par exemple l'exposition Terre natale, ailleurs commence ici, en 2008, conçue en collaboration avec Paul Virilio (1932-2018).

Ce sont donc des thématiques abordées de façon récurrente dans notre programmation, et tout autant par des artistes que des scientifiques. Par ailleurs, l'histoire de la Fondation est très liée aux arbres. En 1994, au moment de son déménagement boulevard Raspail à Paris dans ce bâtiment construit par Jean Nouvel, la Fondation s'est implantée sur l'ancien emplacement de la maison de François-René de Chateaubriand (1768-1848). Juste devant le bâtiment, se trouve un immense cèdre qui a été planté là par l'écrivain en 1822 et autour duquel Jean Nouvel a imaginé son architecture. Tous les jeux de transparence ont été pensés pour donner de l'importance à notre jardin et à tous ces arbres qui entourent la Fondation, qui dialoguent avec l'intérieur du bâtiment et les œuvres exposées.

sc : Quel est le projet global de Nous les arbres?

MP : Le titre de l'exposition Nous les arbres permet de s'interroger sur le lien puissant que l'on peut tisser avec les arbres : comment l'Homme arrive à cohabiter ou, au contraire, à détruire cet univers. Cela permet d'évoquer à la fois ce rapport d'esthétique et d'émerveillement que l'on peut avoir face aux arbres, mais aussi le rapport scientifique, d'étude ou d'observation que l'on peut développer à leur contact.

L'exposition Nous les arbres a pour ambition de redonner la parole aux arbres, de leur rendre la place que l'anthropocentrisme leur a prise depuis toujours. Nous avons constaté que l'on écoute assez peu ces immenses protagonistes du monde vivant.

Nous avons travaillé en collaboration étroite avec le botaniste Francis Hallé qui nous a souvent dit que, si les arbres semblent être immobiles ou statiques, ils sont néanmoins des organismes vivants au sein desquels il se passe énormément de choses ignorées par la communauté des Hommes. Souvent considérés comme des éléments de décor, les arbres sont assez peu considérés comme de véritables êtres vivants. C'est cela qui nous intéressait dans l'exposition : les remettre au cœur de notre relation au vivant. Nous avons ainsi souhaité faire entendre la voix d'une communauté d'artistes, de botanistes, de philosophes, qui développent depuis longtemps un rapport particulier et intime aux arbres.

Nous les arbres, un regard esthétique et scientifique 
L'exposition est à découvrir à la Fondation Cartier à Paris. Elle propose un regard esthétique et scientifique sur les arbres. Le parcours présente les réalisations d'une cinquantaine d'intervenants, artistes contemporains, populations indigènes, botanistes et philosophes qui tous ont développé au cours de leur carrière un lien avec les arbres, mettant ainsi en lumière toute la beauté et la complexité biologique de ces protagonistes du monde vivant, aujourd'hui menacés. Sur deux niveaux et dans le jardin, le parcours de l'exposition déroule trois fils narratifs : celui de la connaissance des arbres, de la botanique à la biologie végétale; celui de leur esthétique, de la contemplation naturaliste à la transposition onirique ; et celui de leur dévastation. Ainsi, cette exposition s'inscrit dans la continuité des expositions de la Fondation Cartier liées aux questionnements sur notre place au sein de la diversité du monde vivant et des cultures, avec notamment la présentation en 2016 du Grand Orchestre des Animaux, inspiré par l'œuvre du bioacousticien Bernie Krause.

(Nous les arbres, du 12 juillet 2019 au 5 janvier 2020 à la Fondation Cartier pour l'art contemporain - 261 boulevard Raspail, 75014 Paris - www.fondationcartier.com)

Le catalogue de l'exposition regroupe en 500 images les œuvres exposées, auxquelles sont associées plusieurs contributions scientifiques et critiques.



$\boldsymbol{s c}$ : Le projet de l'exposition répond à une programmation globale, une demande du public ou un enjeu incontournable?

MP : Un peu aux trois, étant donné que comme je vous le disais, c'est vraiment une thématique qui s'inscrit dans notre programmation depuis des années. Nous essayons en effet de croiser plusieurs disciplines et plusieurs médiums dans les expositions, comme la photographie, l'architecture, les sciences, la philosophie ou les mathématiques. Je ne sais pas s'il existe des équivalents à l'international, mais nous 
travaillons beaucoup avec des institutions dans d'autres pays auxquelles nous prêtons des œuvres de notre collection. Cela nous permet de véhiculer cette dynamique que l'on a insufflée ici à Paris.

$\boldsymbol{s c}$ : Même si vous privilégiez la dimension esthétique, comment ne pas être catastrophiste et, en même temps, ne rien taire?

MP : Cela tient beaucoup à la sélection des œuvres. Cette exposition s'est nourrie des livres que l'on a lus récemment sur l'hypothèse de l'intelligence des plantes, leurs capacités sensibles et sensorielles fascinantes. Nous souhaitions révéler la beauté de ce monde végétal, son extraordinaire intelligence et sa richesse scientifique. Et, par cet émerveillement, ce regard nouveau, nous espérons sensibiliser le visiteur à ce patrimoine, à son rôle et sa fragilité. Et, bien entendu, il était important que le sujet de la déforestation ne soit pas du tout évité et qu'il soit abordé par certains artistes.

Les paysages luxuriants du brésilien Luiz Zerbini, entre jardins botaniques tropicaux et modernité urbaine.

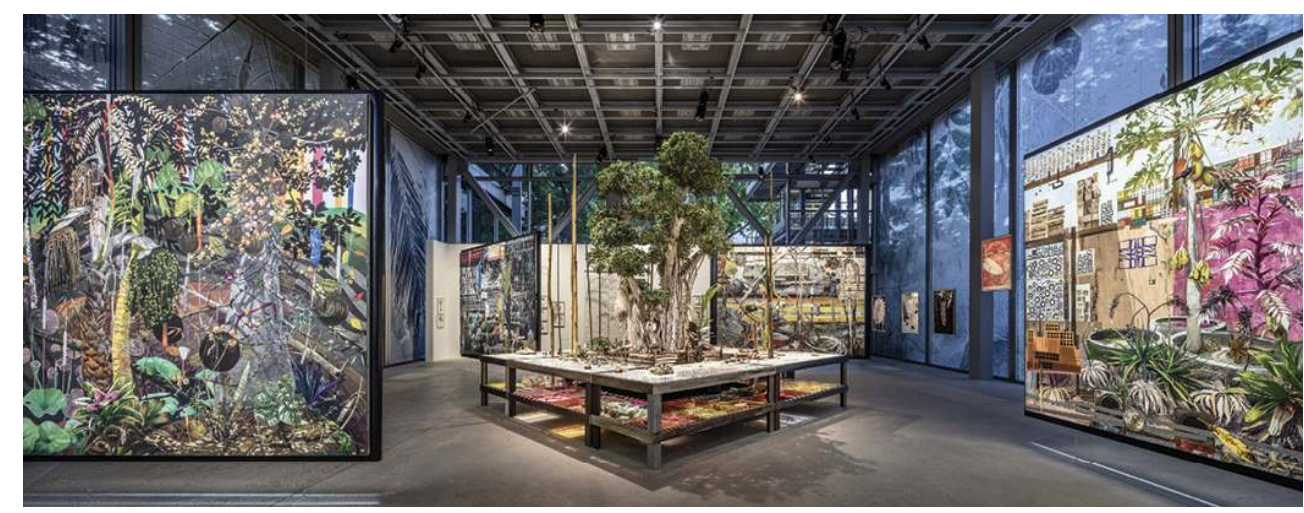

(c) Luc Boegly-Fondation Cartier

sc : Quelles étaient les principales difficultés pour vous, en tant que commissaire, pour traiter cette thématique et faire travailler ensemble artistes et scientifiques ?

MP : Comme je l'ai évoqué, nous insufflons des projets associant anthropologues, scientifiques et artistes depuis longtemps, donc les faire travailler ensemble n'était pas vraiment le cœur du sujet. Ces projets partent de nombreux échanges, de lectures et de travaux conjoints. Une exposition est donc le fruit d'une collaboration, d'une volonté, mais aussi d'une exigence scientifique et d'une certaine rigueur. Nous nous reposons beaucoup sur les scientifiques, sur leurs savoirs et leurs connaissances.

Tout l'enjeu était de travailler sur un sujet d'actualité, un sujet d'une importance majeure parce qu'il porte en lui des considérations politiques contemporaines qui auront un impact déterminant sur notre futur. Finalement, la difficulté réside dans la thématique, dont s'emparent de nombreux artistes aujourd'hui. Nous avons choisi de donner la parole à des artistes et des scientifiques qui tissent un lien fort avec les arbres au sein de leur œuvre et de leur recherche, afin de construire ce projet ambitieux.

sc : Vous avez travaillé à la fois sur le sensible et le scientifique. Dans la première partie de l'exposition, vous êtes sur le sensible, la relation que nous pouvons avoir aux arbres. Puis, 
dans une seconde partie, malgré des dimensions esthétiques fortes, des concepts scientifiques sont abordés.

MP : Tout à fait. Nous avons par exemple décidé de travailler avec Luiz Zerbini qui a réalisé ces immenses peintures orchestrant une rencontre entre des plantes luxuriantes et des signes d'une modernité urbaine, tels que des câbles électriques ou bien des éléments de chantiers de construction. Dans ces toiles, les éléments s'organisent parfois selon une grille géométrique qui évoque aussi la géométrie des villes d'Amérique latine. En associant ces deux typologies, il s'interroge sur la façon dont nous vivons avec les plantes dans la ville, sur la communauté des arbres urbains et leur manière d'exister au sein de ce paysage. Luiz Zerbini questionne aussi, à travers cette installation, la manière dont les plantes et les hommes pourraient cohabiter, en mêlant des dispositifs où les plantes se trouvent tantôt emprisonnées, tantôt libres. Côtoyant cette forêt urbaine, des dessins d'artistes Yanomami sont présentés dans l'exposition. Ce sont des artistes qui vivent au sein de la forêt brésilienne, ils dessinent les arbres qui les entourent afin de rendre compte de leur importance vitale, puisque les arbres leur permettent de se nourrir, de se soigner et d'effectuer des rites chamaniques. Face à eux, des dessins d'artistes Nivaclé du Paraguay sont également présentés. Habitants de la région du Gran Chaco - qui subit actuellement un phénomène de déforestation massif - ces artistes représentent une forêt qui entourait autrefois leurs habitations, peuplée d'animaux et de plantes qui étaient leur source de subsistance.

Parmi les scientifiques avec lesquels nous avons collaboré pour cette exposition, le neurobiologiste Stefano Mancuso, qui dirige le laboratoire international de Neurobiologie végétale de l'université de Florence, a été d'une aide précieuse. Il fut parmi les pionniers de l'hypothèse de l'intelligence des végétaux. Avec lui, nous avons travaillé sur une œuvre présentée dans l'exposition ainsi que sur la chronologie présente dans le catalogue. Nous avons également collaboré avec Emanuele Coccia, philosophe à l'École des hautes études en Sciences sociales (EHESS), qui pense la place des végétaux et des humains au sein du monde des vivants, et s'interroge sur la pertinence de cette place et notre manière de la faire évoluer. 
Symbiosa, l'œuvre de Stefano Mancuso et Thijs Biersteker, « donne la parole aux arbres » grâce à un dispositif numérique qui collecte et interprète les données émises par les arbres.

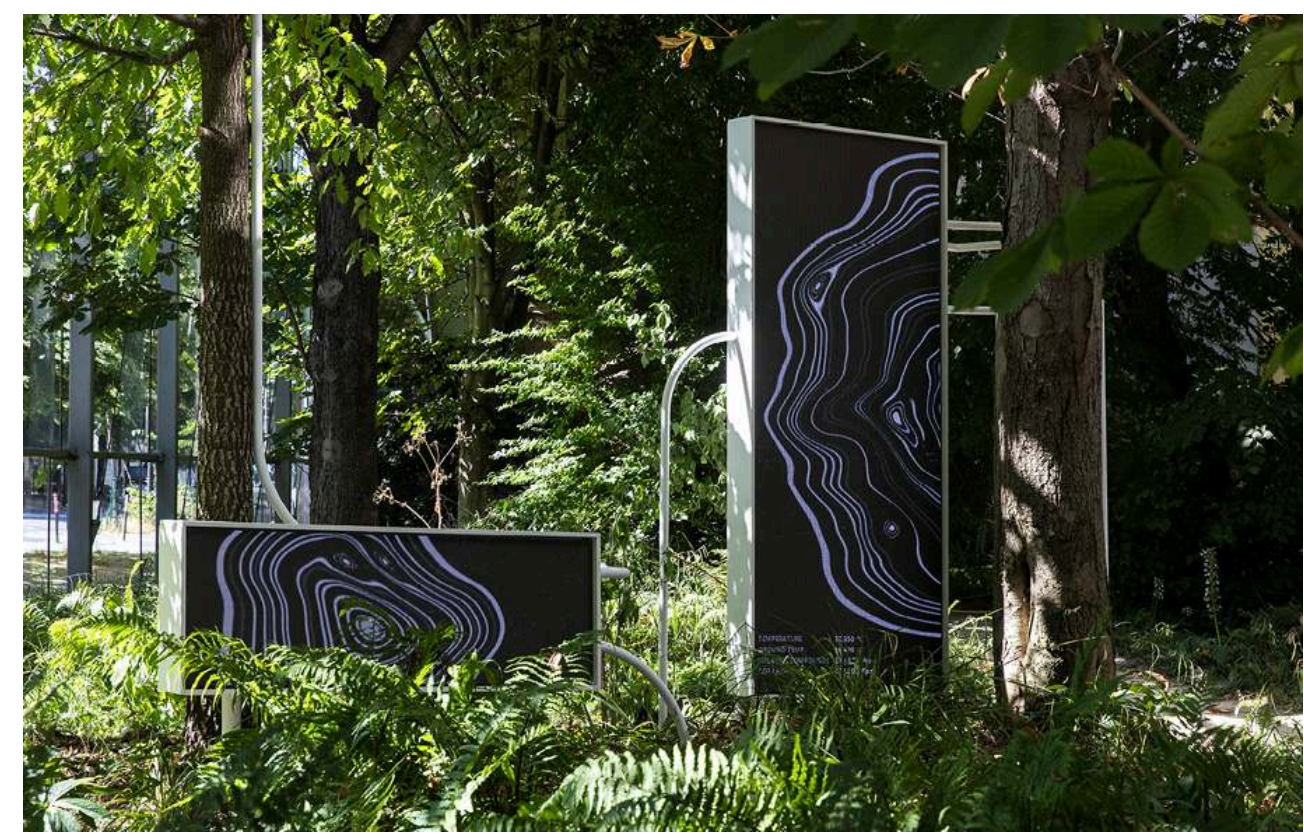

(C) Thibaut Voisin-Fondation Cartier

sc : Pour la mise en exposition de travaux scientifiques, quelles difficultés avez-vous rencontrées?

MP : Si on prend l'exemple de Francis Hallé, cela s'est fait très simplement. Ce botaniste, spécialiste de la canopée et des forêts tropicales, a révolutionné la manière d'appréhender les arbres dans la seconde moitié du XXe siècle. Il a été une grande source d'inspiration pour l'exposition. En plus d'être un grand scientifique, c'est un émerveillé et un amoureux des arbres, un grand dessinateur. Il s'inscrit dans la tradition du dessin botanique depuis le XVIIIe siècle: il explique souvent qu'il a besoin de tourner autour des arbres, de faire des croquis, des petits dessins pour ensuite réaliser le dessin de l'arbre final. Pour lui, la seule façon de pouvoir comprendre les arbres, c'est de les dessiner. Ce rapport-là, qui est à la base scientifique, devient esthétique et poétique, une fois couché sur le papier. Ses dessins et carnets d'expédition présentés dans l'exposition sont donc de véritables œuvres d'art, même si elles partent d'une observation scientifique. Avec lui, nous avons donc effectué une sélection de dessins (parmi 24 000) qui montrent les arbres des différentes régions tropicales dans lesquelles il a voyagé, ainsi que des croquis qui évoquent le Radeau des Cimes ${ }^{1}$, projet initié en 1989.

$\boldsymbol{s c}$ : Le numérique est peu présent dans l'exposition, est-ce un choix délibéré ?

MP : Nous présentons tout de même plusieurs œuvres numériques ou vidéo. Tout d'abord Exit, une œuvre réalisée en 2008 avec Paul Virilio. Cette installation permet, grâce à de la data, de visualiser la déforestation dans trois régions : l'Amazonie, le Cameroun et l'Indonésie. Elle fait un parallèle entre ce processus et la disparition des cultures et des langues dans ces régions. Elle a été actualisée pour la Cop 21, en 2015. Aujourd'hui, les résultats doivent être encore plus édifiants.

Il y a aussi l'œuvre Symbiosia qui résulte d'une collaboration entre Stefano Mancuso et Thijs Biersteker, un artiste néerlandais. Cette œuvre qui se trouve dans le jardin, se 
constitue d'une douzaine de capteurs placés sur deux arbres. Ces capteurs transmettent des informations en temps réel sur deux écrans à Leds disposés aux pieds des arbres. Ainsi, les visiteurs peuvent suivre la réaction des arbres à leur environnement proche, à la pollution, au taux d'ensoleillement, à l'humidité, ou encore à la qualité de l'air de la ville. Cette œuvre permet également de révéler la manière dont les plantes communiquent entre elles.

Nous projetons également deux films très importants. L'un de Raymond Depardon et Claudine Nougaret, Mon Arbre, dresse le portrait d'une dizaine de spécimens (remarquables ou plus familiers filmés sur une place publique, dans la cour d'une ferme ou dans un jardin privé) à travers la parole donnée à des gens qui les côtoient au quotidien. L'autre, dans la dernière salle, est de Paz Encina, une réalisatrice paraguayenne. Il célèbre l'arbre comme lieu d'asile, lieu de mémoire qui nous apporte réconfort et ancrage spirituel.

Nous les Arbres s'organise autourde plusieurs grands ensembles d'œuvres - comme ici celles de Fabrice Hyber - et laisse entendre la voix de ceux qui ont tissé un lien esthétique ou scientifique fort avec les arbres.

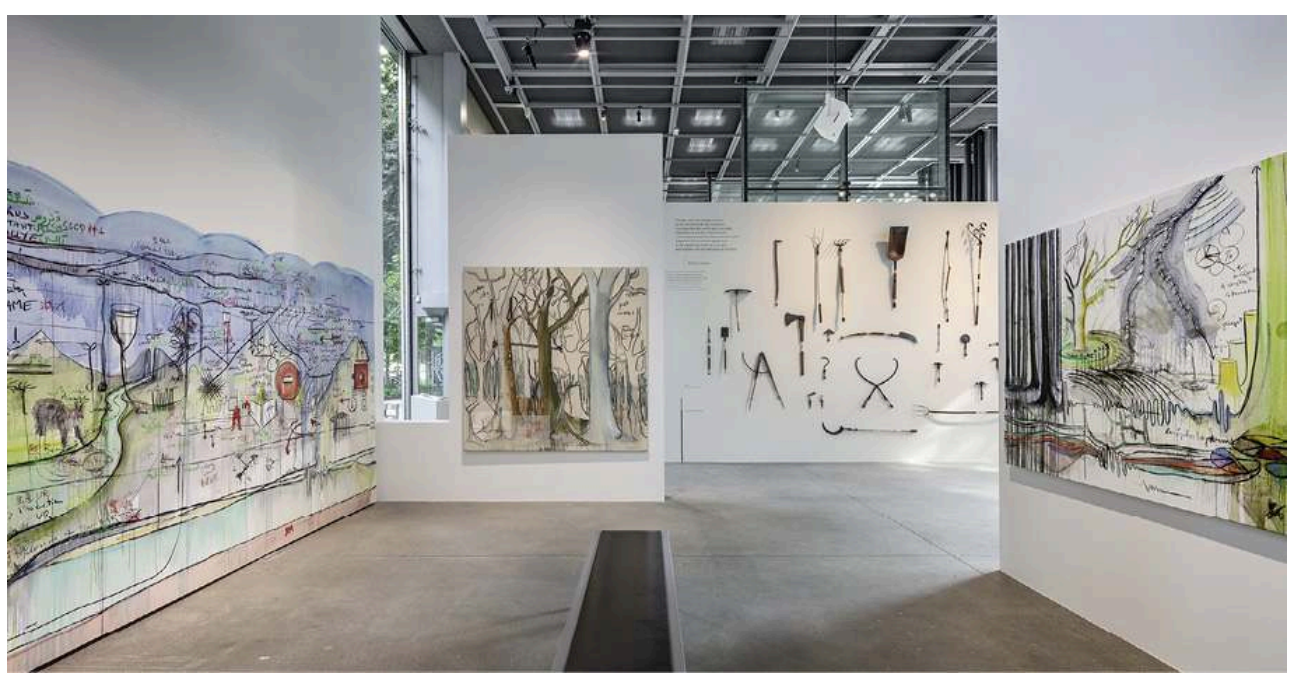

(C) Luc Boegly-Fondation Cartier

sc : Au départ, vous avez visé quel type de public ?

MP : De manière générale, nous visons un grand public et nous accueillons également de nombreux groupes scolaires. La médiation reste très importante. Nous avons un réseau de médiateurs qui travaillent avec nous, qui sont formés par l'équipe curatoriale et qui rencontrent les artistes et les scientifiques avec qui nous élaborons l'exposition. Nous pensons toujours beaucoup nos outils de médiation, de la conception du guide visiteur jusqu'aux différents formats de visites guidées. Et, quels que soient le public et le sujet abordé, nous gardons la même rigueur artistique et scientifique.

Nous avons des retours de manière informelle, grâce à une tablette numérique sur laquelle le public note régulièrement des commentaires. Les réactions sont majoritairement positives : les visiteurs soulignent l'aspect esthétique de l'exposition et du lieu. Nous avons d'ailleurs beaucoup travaillé sur le lien entre les espaces d'exposition et le jardin, en jouant sur la transparence du bâtiment, pour faire de notre jardin - où il est si agréable de se promener - une cinquième salle d'exposition. 
Nous observons par ailleurs que le sujet de l'exposition touche et interroge beaucoup. Aujourd'hui, nous avons en effet dépassé les 150000 visiteurs, ce qui est énorme. Cela témoigne de la qualité de l'exposition et de l'importance d'un tel sujet aujourd'hui.

\section{NOTES}

1. Site Web de l'opération Canopée, connue également sous le nom de Radeau des Cimes: www.radeau-des-cimes.org

\section{RÉSUMÉS}

Depuis plusieurs années, la Fondation Cartier, à Paris, réunit artistes contemporains et chercheurs dans le cadre d'expositions qui abordent les relations entre l'Homme et son environnement. Commissaire associée de l'exposition Nous les arbres, Marie Perennes évoque avec Samuel Cordier ce projet original qui, par une approche esthétique, vise à sensibiliser les visiteurs à l'importance et à la place des arbres au sein de la communauté des vivants.

\section{INDEX}

Mots-clés : développement durable, exposition, organisation culturelle (hors musées)

\section{AUTEURS}

\section{MARIE PERENNES}

Conservatrice et commissaire d'exposition à la Fondation Cartier pour l'art contemporain marie.perennes@fondation.cartier.com

\section{SAMUEL CORDIER}

Directeur de l'Ocim

samuel.cordier@u-bourgogne.fr 